\title{
Effect of ultrafine poly( $\varepsilon$-caprolactone) fibers on calcium phosphate cement: in vitro degradation and in vivo regeneration
}

This article was published in the following Dove Press journal:

International Journal of Nanomedicine

7 January 2016

Number of times this article has been viewed

\author{
Boyuan Yang' \\ Yi Zuo' \\ Qin Zou' \\ Limei $\mathrm{Li}^{\prime}$ \\ Jidong $\mathrm{Li}^{1}$ \\ Yi Man² \\ Yubao $\mathrm{Li}^{1}$ \\ 'Research Center for Nano \\ Biomaterials, Analytical and Testing \\ Center, Sichuan University, Chengdu, \\ People's Republic of China; ${ }^{2}$ State \\ Key Laboratory of Oral Disease, \\ West China Hospital of Stomatology, \\ Sichuan University, Chengdu, People's \\ Republic of China
}

Correspondence: Yi Zuo; Yubao Li

Research Center for Nano Biomaterials, Sichuan University, 29 jiuyanqiao

Wangjiang Road, Chengdu 610064,

People's Republic of China

Tel +862885418178

Fax +862885417273

Email zoae@scu.edu.cn;

nic7504@scu.edu.cn

\begin{abstract}
We incorporated ultrafine polymer fibers into calcium phosphate cement (CPC) to improve the resorption rate of $\mathrm{CPC}$ with fiber degradation. Different weight percentages of electrospun poly( $\varepsilon$-caprolactone) fibers $(0 \%, 3 \%$, and $7 \%$, named as ultrafine fiber-incorporated CPC0 [UFICPC0], UFICPC3, and UFICPC7) were included into preset CPC specimens for in vitro immersion in lipase phosphate-buffered solution and long-term in vivo implantation in the femoral condyle of rabbits. The effect of the ultrafine poly( $\varepsilon$-caprolactone) fibers with a diameter ranging from nanometer to micrometer on CPC degradation was evaluated by measuring the $\mathrm{pH}$ of the medium, mass loss, porosity, and physiochemical properties. For the in vivo evaluation, histomorphometrical analysis as well as three-dimensional (3D) reconstruction was applied to assess the osteogenic properties of the CPC composite. After in vitro immersion and in vivo implantation, the total porosity and macroporosity as well as the bone formation and ingrowth increased significantly during time in the fiber-incorporated CPC specimens. After 24 weeks of implantation, the degraded space was occupied by newly formed bone, and the UFICPC3 and UFICPC7 composites showed a $\sim 3.5$ times higher fraction of bone volume than that of the pristine CPC (UFICPC0). In vitro and in vivo results proved that the introduction of ultrafine degradable fibers within a CPC matrix can be used to improve macroporosity efficiently and enhance CPC degradation and bone ingrowth largely.
\end{abstract}

Keywords: ultrafine fibers, calcium phosphate cement, macroporosity, degraded space, bone ingrowth

\section{Introduction}

Calcium phosphate cements (CPCs) have gained clinical acceptance to repair a bone defect due to their recognized bioactivity and moldability. ${ }^{1}$ Typical applications are the treatment of maxillofacial defects or deformities and augmentation of spine. ${ }^{2}$ The material can even be applied by using minimally invasive surgery and for the filling of complex-shaped defects. ${ }^{3}$ However, two major drawbacks limit their clinical use. One is the inherent brittleness derived from the composition and microstructure. ${ }^{4}$ The other is the slow resorption rate. ${ }^{5,6}$

The incorporation of bioresorbable or biodegradable materials into the $\mathrm{CPC}$ matrix can circumvent these two drawbacks. Fiber reinforcement of CPC is the most successful approach to toughen this brittle material. ${ }^{1}$ Various fibers have already been used for such a purpose, such as ceramic fibers (eg, carbon or glass fibers), nonresorbable polymer fibers, and resorbable polymer fibers (eg, polylactide, polyglycolide/polylactide copolymers, poly- $\varepsilon$-caprolactone [PCL], etc). ${ }^{7}$ When bioresorbable or biodegradable fibers are used, this will not only contribute to the initial mechanical strength, but also 
porosity will be created after fiber degradation. The porosity will increase the surface area of CPC matrix, resulting in increased resorption of the cement and facilitation of bone ingrowth.

Most of the articles dealing with degradable fiberreinforced CPC focus on the mechanical properties and cell response. Only a few report about the long-term in vivo behavior. ${ }^{8-10}$ The results of these studies are contradictory on the efficient of degradable fibers addition. For example, in a 52-week study dealing with poly(lactic-co-glycolic acid) (PLGA) fiber-reinforced CPC, fiber degradation could not be associated with a promising bone regeneration. ${ }^{9}$ In another in vivo study, a porous material transferred from the $\mathrm{CPC} /$ PLGA composite exhibits a perfect osteoconduction accompanied with osteoblast-like cells and Haversian canals after 24-week implantation. ${ }^{10}$ Therefore, further in vivo studies should be carried out to obtain more data on degradation and bone regeneration of fiber-reinforced CPC.

Besides commercial fibers in the range of $10-120 \mu \mathrm{m}$, ultrafine electrospun fibers $(0.5-2 \mu \mathrm{m})$ have been used to build scaffolds for bone tissue engineering to mimic the nanofibrous collagen network of the bone matrix. ${ }^{11}$ Such ultrafine fibers have several attractive characteristics, as higher specific surface area and superior mechanical performance, compared with any other regularly formed material. ${ }^{12}$ However, a scaffold prepared from such fibers is not rigid enough to withstand the mechanical stresses during bone tissue neogenesis. Therefore, in a previous study, we prepared and evaluated a series of CPC composites reinforced with electrospun biodegradable fibers on mechanical properties, porosity, and morphological structure. ${ }^{13}$ The results indicated that the incorporation of ultrafine fibers enhanced the fracture resistance and porosity of CPC. In particular, the work of fracture of CPC composites with $7 \mathrm{wt} \%$ PCL fibers increased dramatically, which is approximately 30 times higher than that of pristine CPC.

In this study, we aim to further investigate the effects of ultrafine PCL fibers on their biodegradation and osteogenetic properties by both in vitro and in vivo evaluation. For the in vitro degradation assay, enzyme containing medium was used to partly mimic the physiological environment. The effect of the PCL fibers with a diameter ranging from the nanometer to micrometer scale on CPC degradation was evaluated by measuring the $\mathrm{pH}$ of the medium, mass loss, porosity, and physiochemical properties. For the in vivo evaluation, histomorphometrical analysis as well as three-dimensional (3D) reconstruction were applied to assess the bioresorption and the bone regeneration of the composite materials.

\section{Materials and methods Materials}

The CPC powder $\left(\mathrm{Calcibon}^{\circledR}\right)$, consisting of $61 \% \alpha$-tricalcium phosphate (TCP), 26\% $\mathrm{CaHPO}_{4}, 10 \% \mathrm{CaCO}_{3}$, and $3 \%$ precipitated hydroxyapatite (HA), was provided by Biomet Merck (Berlin, Germany). The cement liquid applied was a $1 \%$ aqueous solution of $\mathrm{Na}_{2} \mathrm{HPO}_{4}$. PCL with an average molecular weight of 80,000 was purchased from SigmaAldrich (St Louis, MO, USA). Lipoprotein lipase (Lipase PS Amano SD, Lot TLK0015, Wako Pure Chemical Industries, Ltd, Osaka, Japan) was dissolved in phosphate buffer solution (PBS) $(\mathrm{pH}=7.4)$ to enzymatically degrade PCL fibers and CPC composites.

\section{Preparation of PCL fiber bundles}

Ultrafine PCL fibers were fabricated by electrospinning using a special water pool technique as previously described. ${ }^{13}$ Briefly, PCL granules were dissolved in tetrafluoroethylene to obtain $12 \%(\mathrm{w} / \mathrm{v})$ polymer solution under gentle stirring for 12 hours. A commercially available electrospinning apparatus (Advanced Surface Technology, Bleiswijk, the Netherlands) was used for the fiber bundles fabrication. Upon applying a high voltage of $18.15 \mathrm{kV}$, PCL solution was ejected from a needle (inner diameter of $0.8 \mathrm{~mm}$ ) by feeding from a glass syringe at a rate of $1.5 \mathrm{~mL} / \mathrm{h}$. The polymer jet was directed to a grounded water pool positioned under the needle at a distance of $28 \mathrm{~cm}$, and the charged polymer fibers were deposited on the surface of water. Then, the random fibers were drawn to a rotating mandrel (diameter of $4.5 \mathrm{~mm}$ ) at a speed of $12 \mathrm{rpm}$ to form an oriented fiber bundle (diameter of single fibers were ranging from 400 to $1,100 \mathrm{~nm})$. The collected fiber bundles $(\Phi 0.2 \mathrm{~mm})$ were freeze-dried for 24 hours, then cut into pieces of $3 \mathrm{~mm}$ in length, and stored at $-20^{\circ} \mathrm{C}$.

\section{Preparation of cement/fiber composites}

CPC powder and $1 \%(\mathrm{w} / \mathrm{v}) \mathrm{Na}_{2} \mathrm{HPO}_{4}$ solution were mixed inside a $2 \mathrm{~mL}$ plastic syringe and subjected to a vigorous shaking for 15 seconds (YDM mixing apparatus, Hangzhou Xinya Co. Ltd, Hangzhou, People's Republic of China). A liquid/powder (L/P) ratio of 0.33 was applied. After shaking, the mixture in a paste form was immediately injected into a container and blended manually by a spatula with the cut electrospun fiber bundles. The weight fractions of the fibers were $0 \%, 3 \%$, and $7 \%$, respectively. Then, the fiberpaste mixture was filled into a Teflon mold of $\Phi 5 \times 5 \mathrm{~mm}^{3}$ and gently pressed with a spatula. The composite mixture in the mold was covered with a glass slide and stored at $37^{\circ} \mathrm{C}$ 
in a humidor for 24 hours. Then the hardened composite specimens were taken out from the mold and vacuum dried for testing.

For convenience, the ultrafine fiber-incorporated CPC (UFICPC) with fiber weight fractions of $0 \%, 3 \%$, and $7 \%$ were named as UFICPC0, UFICPC3, and UFICPC7, respectively. The fiber volume of UFICPC3 and UFICPC7 composites has been calculated by:

$$
\nu \%=\frac{\rho_{\mathrm{HA}} \times \mathrm{wt} \%}{\rho_{\mathrm{PCL}}},
$$

where $\rho_{\mathrm{HA}}=3.156 \mathrm{~g} \cdot \mathrm{cm}-3, \rho_{\mathrm{PCL}}=1.145 \mathrm{~g} \cdot \mathrm{cm}-3, v$ means fiber volume, wt means fiber weight.

\section{In vitro degradation}

For the enzymatic degradation studies, three groups of preset UFICPC specimens (UFICPC0, UFICPC3, and UFICPC7) and electrospun PCL fibers (100 mg each) were immersed in a vial filled with a lipase solution consisting $5 \mathrm{~mL}$ PBS ( $\mathrm{pH}=7.4$ ) with 200 unit/mL lipoprotein lipase (Lipase PS, Wako Pure Chemical Industries, Ltd) for up to 7 days. The vials were placed in water bath thermostated at $37^{\circ} \mathrm{C} \pm 0.2^{\circ} \mathrm{C}$. At each time point, specimens were removed out and fully dried in a vacuum oven until weight constancy after removal from the incubation medium.

\section{$\mathrm{pH}$ measurement}

The $\mathrm{pH}$ value of residual solution in each vial was collected and measured by $\mathrm{pH}$ meter $(\mathrm{n}=3)$ at each timepoint, referring to the earlier study for degradation of PCL, ${ }^{14,15}$ to observe whether the acidic compounds degraded from PCL would cause the condition change of CPC hydrolysis and degradation.

\section{Morphology observation}

Before degradation, porous structure and fiber distribution of specimens were visualized by microcomputed tomography (micro-CT) ( $\mu \mathrm{CT} 100$, SCANCO Medical AG, Brüttisellen, Switzerland) at a resolution of $2.4 \mu \mathrm{m}$ per pixel. Acquisition parameters for the scanning were $70 \mathrm{kV}$ and $85 \mu \mathrm{A}$. The region of interest (ROI) in which the porosity was measured and defined as a cylindrical area covering preset specimen of a $\Phi 5 \times 5 \mathrm{~mm}^{3}$. The morphology of blade-cut cross-section of specimens before mixed (scanning electron microscope [SEM], Jeol 6310, JEOL Ltd, Tokyo, Japan) and after composite degradation for 1,3 , and 7 days was observed using a SEM (JSM-6500LV, JEOL Ltd). Samples were sputtercoated with gold prior to observation.

\section{Mass loss test}

In order to characterize the degradability, the composite weight was measured after removal from the immersion medium, and vacuum dried over 1 week at $37^{\circ} \mathrm{C}$. Mass loss was determined using Equation 2:15

$$
R_{\mathrm{L}}=\frac{m_{0}-m_{n}}{m_{0}} \times 100 \%,
$$

where $R_{\mathrm{L}}$ is the mass loss of the sample (\%) on $t=n, m_{0}$ is the mass of the sample $(\mathrm{g})$ on $t=0, m_{n}$ is the mass of the sample (g) when $t=n$.

\section{Porosity determination}

The porosity of preset CPC specimens containing PCL fibers was determined according to the method described previously. ${ }^{13}$ In brief, the UFICPC composites were weighed before and after burning out PCL fibers at $400^{\circ} \mathrm{C}$ for 2 hours. Equations 3 and 4 were used for the calculation of the total porosity and the macroporosity:

$$
\begin{aligned}
\mathcal{E}_{\mathrm{tot}, \mathrm{n}} & =\frac{1-m_{\text {burnt,n }}}{V \times \rho_{\mathrm{HA}}} \times 100 \% \\
\mathcal{E}_{\text {macro,n }} & =\frac{1-m_{\text {burnt }, \mathrm{n}}}{m_{\text {micro, } \mathrm{n}}} \times 100 \%,
\end{aligned}
$$

where $\varepsilon_{\text {tot,n }}$ is total porosity ( $\left.\%\right)$ when $t=\mathrm{n}, \varepsilon_{\text {macro,n }}$ is macroporosity $(\%)$ when $t=\mathrm{n}, m_{\text {burnt,n }}$ the mass (g) of UFICPC composite after burning when $t=\mathrm{n}, V$ is the volume of the sample $\left(\mathrm{cm}^{3}\right), \rho_{\mathrm{HA}}$ is the density of HA $\left(3.156 \mathrm{~g} / \mathrm{cm}^{3}\right), m_{\text {micro,n }}$ the average mass (g) of the pristine CPC sample after burning when $t=\mathrm{n}$. Tests were performed triplicate.

\section{Phase composition analysis}

Three groups of UFICPC samples before degradation as well as after degradation for 1 and 7 days were ground for testing. The structure of the CPC composites was studied by X-ray diffraction (XRD, DX2500, Dandong Fangyuan Instrument LLC, Dandong, People's Republic of China) to determine the phase composition, relative intensity of crystalline PCL in composites, and the phase conversion of CPC. The X-ray generator was operated at a voltage of $40 \mathrm{kV}$ with a tube current of $25 \mathrm{~mA}$. The diffraction pattern was recovered by a proportional counter in the angular range from $2 \theta=10^{\circ}$ to $70^{\circ}$ 
using a step width of $\Delta(2 \theta)=1^{\circ}$. The same ground samples were also used for Fourier transform infrared spectroscopy (FTIR, Nicolet 6700, Thermo Fisher Scientific, Waltham, MA, USA). The typical PCL absorption band and calcium phosphate band of the UFICPC composite were measured over a wavenumber range of $400-4,000 \mathrm{~cm}^{-1}$ with a $\mathrm{KBr}$ sample weight ratio of 100/1.

\section{In vivo experiment and evaluation}

The animal experiment was approved by the Animal Ethical Committee of Sichuan University and performed as per the China national guidelines for the care and use of laboratory animals. Thirty-six healthy adult New Zealand White rabbits (18 males and 18 females), weighing $2.2-3.0 \mathrm{~kg}$, were divided randomly into three experimental groups. Anesthesia was induced by ear vein injection of 3\% sodium pentobarbital at a dose of $1 \mathrm{~mL} / \mathrm{kg}$. The hind limbs of rabbits were shaved, washed, and disinfected with povidone-iodine. A cylindrical bone defect (diameter $5.5 \mathrm{~mm}$, depth $5 \mathrm{~mm}$ ) was drilled on the left side after exposure of the distal femoral condyle.

Specimens of UFICPC0, UFICPC3, and UFICPC7 were implanted into the created condylar defects of three experimental groups, respectively. Postoperative animals were allowed freedom of movement, and daily intramuscular injection of penicillin and gentamicin was given for 3 days to prevent infection. The implantation periods were set for 4,8 , 12, and 24 weeks. At each time point, three rabbits were in each group. At each timepoint of implantation, rabbits were sacrificed with an overdose of anesthesia and samples of 4 , 8 , and 12 weeks were harvested for histological evaluation. Only the harvested samples of 24 weeks were evaluated by micro-CT analysis.

\section{Microcomputed tomography analysis}

Three-dimensional analysis was conducted on the 24 samples using a vivaCT40 micro-CT imaging system (SCANCO Medical AG). In brief, each sample was placed with its long axis in the vertical position, and immobilized with a foam pad in a cylindrical sample holder for scanning. The femoral condyles of each sample were scanned. A ROI of $5 \mathrm{~mm}$ in diameter was defined to cover the created defect. The same ROI was used in all the samples. The scanning system was set at $70 \mathrm{kV}, 114 \mu \mathrm{A}$, and $8 \mathrm{~W}$; a total of 300 microtomographic slices with a slice increment of $20 \mu \mathrm{m}$ were taken for each sample. 3D Gaussian filter was constrained at $\sigma=1.2$ and support $=2$ for partial suppression of the noise in the volumes. A threshold equal to or above 401 represented the $\mathrm{CPC}$ component; a threshold below 401 and equal to or above 270 signified the bone tissue; a threshold below 270 represented the marrow and soft tissue. ${ }^{16,17}$ The $3 \mathrm{D}$ reconstructed images were acquired from a series of 130 serial 2,048 $\times 2,048$ bitmap images. In addition to the visual assessment of structural images of bone and cement, morphometric parameters were determined from the microtomographic datasets using direct 3D morphometry, including the fraction of bone volume/ tissue volume (BV/TV), material volume (MV)/TV, and trabecular number $(\mathrm{Tb} . \mathrm{N})$.

\section{Histological examination}

During the study, the tissue-covered specimens were retrieved and fixed in $10 \%$ formalin. Preliminarily, one series of specimens at different interval times were decalcified in buffered formic acid for 7 days at room temperature and embedded in paraffin. After the paraffin-embedded specimens were cut into slices and stained with hematoxylin/eosin, the decalcified sections were observed under optical microscope. Due to the fact that the major components of testing materials were inorganic CPC, the implant sections were fragmented and could not provide enough information of the study. Hence, only two serial specimens were dealing with embedding undecalcified bone method for further histological investigation. In this regard, the statistical analysis of animal testing could not be practiced without enough samples in this paper.

Therefore, the harvested samples of 4,8 , and 12 weeks were fixed in 4\% formaldehyde for 1-2 weeks, then dehydrated in graded ethanol solution, and embedded in methylmethacrylate. Nondecalcified cross-sections of the embedding samples were prepared using Leica SP1600 hard tissue slicer. The ultrathin sections $(\sim 100 \mu \mathrm{m})$ were stained with methylene blue and basic fuchsin and examined via a light microscope.

\section{Statistical analysis}

SPSS software was used to establish a database and statistical analysis, the data from in vitro test were expressed as mean \pm standard deviation. Statistical significance was determined by the $P$-value of Student's $t$-test, $P<0.05$ indicates statistically significant. The data for one-way analysis of variance were analyzed via a Tukey multiple comparison test.

\section{Results \\ Effect of enzymatic degradation on $\mathrm{pH}$ value in vitro}

The $\mathrm{pH}$ values of the enzymatic media were measured at different immersion times, as shown in Figure 1. The release of 

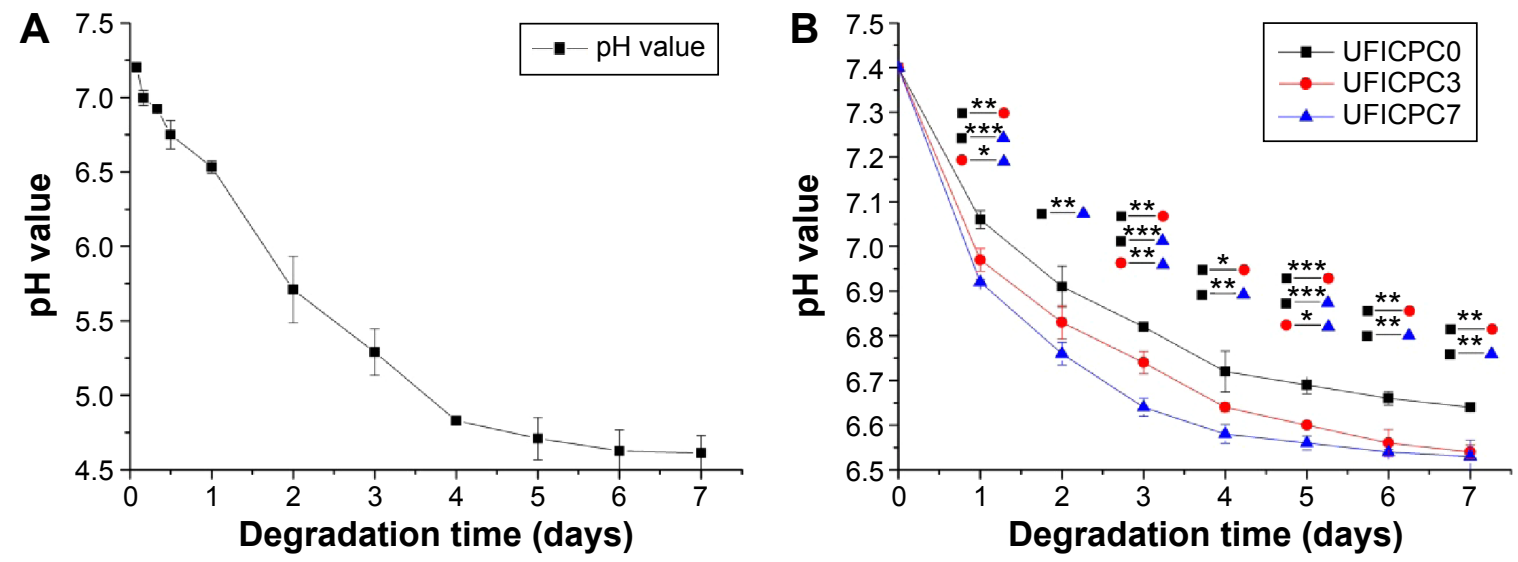

Figure I Effect of fiber and cement degradation on $\mathrm{pH}$ value of lipase PBS.

Notes: (A) Pure ultrafine PCL fibers, the pH value of the medium rapidly decreased to lower than 4.8 after 4 days; (B) composites of UFICPC0, UFICPC3, and UFICPC7. The UFICPC composites with or without $P C L$ fibers showed a similar trend, but with a relatively slower rate. Significantly different at $* P<0.05$, $* * P<0.0$ I, $* * * P<0.001$. Abbreviations: CPC, calcium phosphate cement; PBS, phosphate buffer solution; PCL, poly( $\varepsilon$-caprolactone); UFICPC, ultrafine fiber-incorporated CPC.

acidic products from the pure PCL fibers in the medium results in a faster decrease of $\mathrm{pH}$ value from 7.4 to 4.8 in 4 days (Figure 1A). Thereafter, the $\mathrm{pH}$ value decreased slowly to 4.5 at day 7. The UFICPC composites with or without ultrafine PCL fibers showed a similar trend, but with a relatively slower rate, ie, the $\mathrm{pH}$ on day 7 is still above 6.5 (Figure 1B). The $\mathrm{pH}$ values of the enzymatic media for the ultrafine fiberreinforced composites (UFICPC3 and UFICPC7) are lower than the pristine CPC (UFICPC0). At 1, 3, and 5 days of incubation, the $\mathrm{pH}$ values of the enzymatic medium for the 3 and $7 \mathrm{wt} \%$ fiber fractions differed significantly $(P<0.05)$, but this difference did not exist at day 6 and 7 .

\section{Effect of enzymatic degradation on morphology and porosity}

Cross-sectional micro-CT images were obtained from all materials (Figure 2B-D). Small air bubbles or small pores were visible as black color in the materials. Density morphology was presented in the UFICPC0 sample (Figure 2B). Ultrafine PCL fibers in the UFICPC 3 and UFICPC7 cement could be clearly recognized (dark gray-black elongated structures) and also some irregular-shaped cracks (black color) were observed (Figure 2C and D). Interconnected and tangled fiberlike morphologies dispersed in the compact cement matrix. Table 1 lists fiber volumes and porosities of composites that have been calculated from different theories.

The SEM micrographs in Figure 2A show a fibrous structure with a diameter ranging from nanometer to micrometer, and Figure 3 demonstrates a progressive fiber degradation of the composites in the lipase PBS. The UFICPC0 specimens showed the presence of some surface microporosity. No apparent degradation of the UFICPC0 specimens was observed. On the other hand, it seemed that some additional deposition of material occurred during incubation for the chemical conversion of calcium and phosphate ions (Figure 3A-D). For sample UFICPC3 and UFICPC7 specimens, besides conversion of the CPC matrix, the PCL fibers became thinner from micrometer to nanometer with time and a lot of fibers had completely disappeared on day 7 , leaving many fiber traces (grooves) in the CPC matrix (Figure 3, E-H for UFICPC3, I-L for UFICPC7).

The weight change of pure PCL fibers and UFICPC cements in lipase PBS solution is plotted in Figure 4. Pure PCL fibers degraded faster in the enzymatic solution than in pure PBS. The mass loss increased rapidly with time and reached approximately $55 \%$ on day 7 (Figure $4 \mathrm{~A}$ ). The UFICPC0 samples (pristine CPC cement) showed a slight weight increase during incubation (Figure 4B, $0 \mathrm{wt} \%$ curve), indicating a deposition of some component in enzymatic PBS solution on the CPC matrix. The UFICPC3 and UFICPC7 specimens showed a linear decrease in mass after 1 day of incubation, but mass loss was much lower compared with pure PCL fibers. Compared to UFICPC3, UFICPC7 specimens showed a significantly higher mass loss $(P<0.05 \%)$.

By means of the burning-out method, ${ }^{15}$ the total porosity and macroporosity of composite cements at different immersion times are calculated and shown in Figure 5. It can be seen that both the total porosity and macroporosity increase with the immersion time, sample UFICPC7 holds higher porosities than samples UFICPC3 and UFICPC0. Significant difference in microporosity is present between UFICPC7 and UFICPC3, and among UFICPC7, UFICPC3, and UFICPC0 in total porosity. After immersion, the macroporosities of UFICPC3 and UFICPC 7 change from starting $10.23 \%$ and $19.21 \%$ to final 

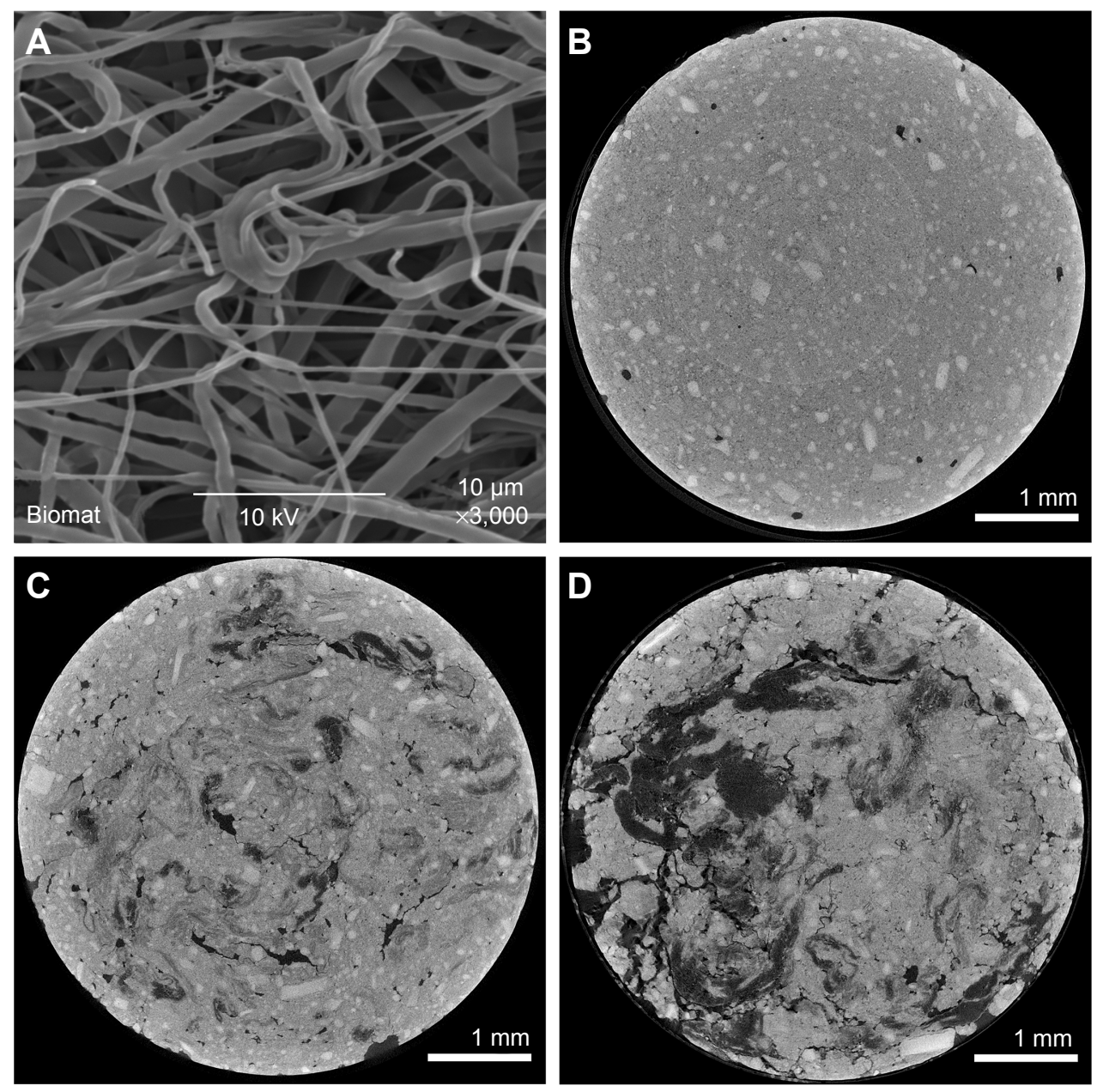

Figure 2 Morphology observation of ultrafine PCL fibers and UFICPC composites.

Notes: (A) SEM micrographs of electrospun ultrafine fibers, which were prepared from I2\% (w/v) PCL solutions and collected by water pool with a nano to micro fibrous structure. Micro-CT cross-sectional images of sample UFICPC0 (B), UFICPC3 (C), and UFICPC7 (D). Black colored area was distinguished as small air bubbles or small pores in the CPC by micro-CT machine. Fiber-like morphologies in the UFICPC 3 and UFICPC7 cement could be clearly recognized as dark gray - black elongated structures. Irregular-shaped black colored area indicates cracks between fibers and cement.

Abbreviations: CPC, calcium phosphate cement; CT, computed tomography; PCL, poly(E-caprolactone); SEM, scanning electron microscope; UFICPC, ultrafine fiberincorporated CPC.

$15.0 \%$ and $24.5 \%$ on day 7 , which is almost two times higher than the calculated fiber volume ( $8.27 \%$ and $12.29 \%)$.

\section{Effect of enzymatic degradation on phase composition}

The XRD patterns in Figure 6B and $\mathrm{C}$ show that the monetite and $\alpha$-TCP phase composition in UFICPC 3 and UFICPC 7 converts progressively into apatite within 7 days, which is faster than the conversion of pristine CPC (Figure 6Bf and $\mathrm{Ci}$ vs Ac). After 7 days, both the monetite and $\alpha$-TCP components can still be observed in UFICPC0 (Figure 6Ac). ${ }^{15,18}$ As the content of ultrafine PCL fibers is very small ( 3 and $7 \mathrm{wt} \%$ ), only a weak characteristic peak of PCL component located approximately $22^{\circ}$ can be found in samples UFICPC 3 and UFICPC7. This peak becomes weaker or disappears completely during incubation from day 1 to 7 in lipase PBS.

Table I Fiber volumes and porosities of composites by theoretical calculation and micro-CT

\begin{tabular}{llllll}
\hline Specimens & \multicolumn{2}{l}{ Theoretical calculation } & & \multicolumn{2}{l}{ Calculation by Micro-CT } \\
\cline { 2 - 3 } \cline { 6 - 7 } & Fiber content (wt\%) & Fiber volume (vol\%) & & Macroporosity (\%) & Total porosity (\%) \\
\hline UFICPC0 & 0 & 0 & 0.42 & 1.17 \\
UFICPC3 & 3 & 8.27 & 5.14 & 6.69 \\
UFICPC7 & 7 & 12.29 & 11.39 & 18.86 \\
\hline
\end{tabular}

Note: The calculated data from micro-CT were different with theoretical calculation.

Abbreviations: CPC, calcium phosphate cement; CT, computed tomography; UFICPC, ultrafine fiber-incorporated CPC. 

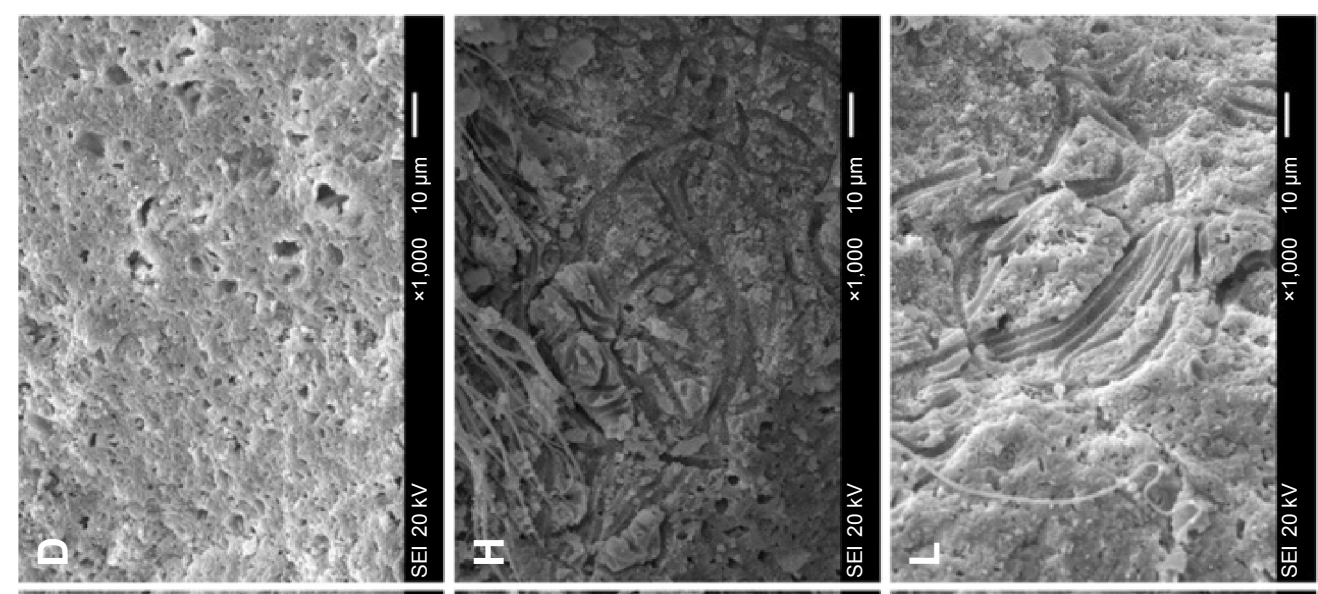

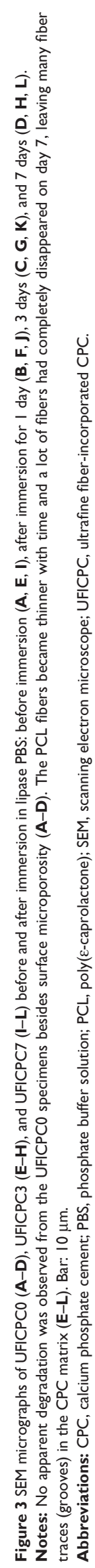


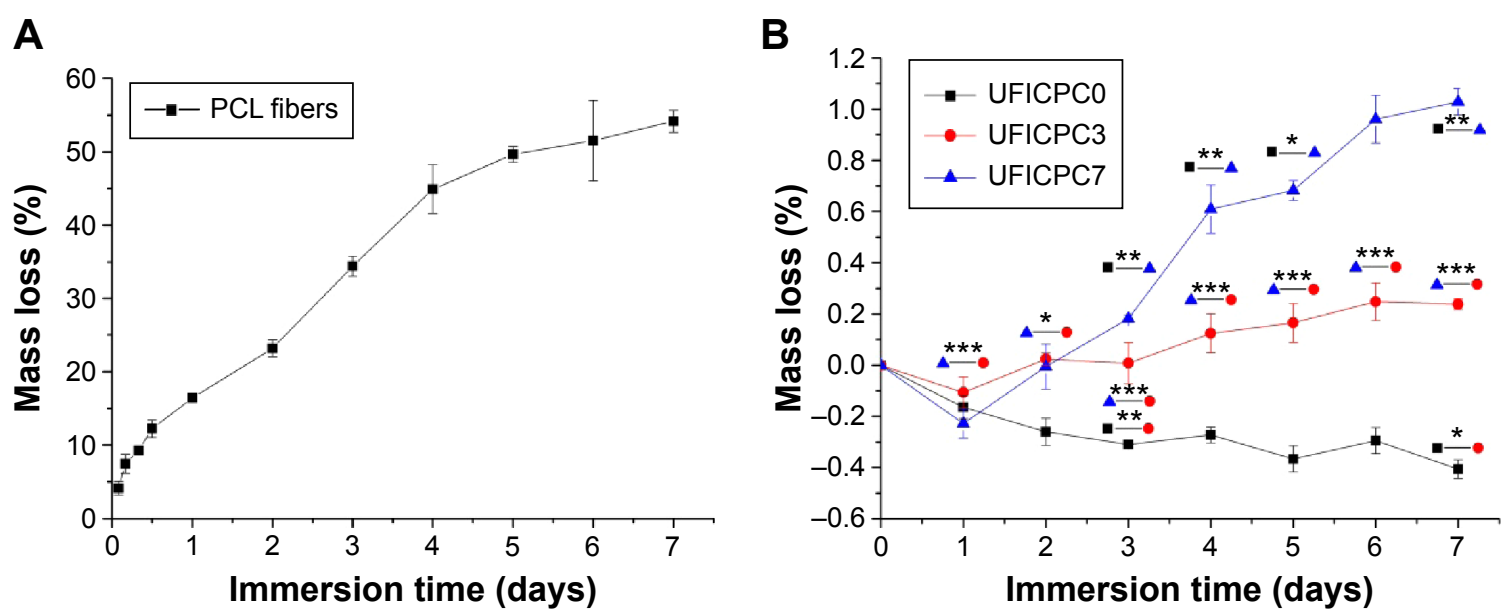

Figure 4 Weight change of pure PCL fibers (A) and UFICPC cements (B) with immersion time.

Notes: Pure PCL fibers degraded fast in the enzymatic solution (A). UFICPC0 increased slightly indicating a deposition process during incubation. Other composites (UFICPC3 and UFICPC7) showed a linear decrease in mass after I day incubation. Significantly different at $* P<0.05, * * P<0.01, * * * P<0.001$.

Abbreviations: CPC, calcium phosphate cement; PCL, poly(E-caprolactone); UFICPC, ultrafine fiber-incorporated CPC.

Figure 7 shows the infrared spectra of three UFICPC composites before and after immersion in enzymatic PBS. The chemical groups of pristine CPC cement (UFICPC0) do not show an obvious change after immersion for 1 and 7 days (Figure 7A). For UFICPC3 and UFICPC7 composites (Figure 7B and C), the peak at 1,729 $\mathrm{cm}^{-1}$ represents the amorphous phase of PCL, and the peaks at 1,298, 1,192, and $735 \mathrm{~cm}^{-1}$ represent the crystalline phase of PCL. The peak at $1,729 \mathrm{~cm}^{-1}$ corresponds also to the $\mathrm{C}=\mathrm{O}$ stretch of $\mathrm{PCL}$ and decreases significantly in intensity during incubation time, which indicates a preferred enzymatic degradation of the amorphous PCL segments. All other peaks are similar to pristine CPC cement and correspond with the phosphate, hydroxyl and carbonate groups. The set CPC matrix contains a carbonated HA structure.

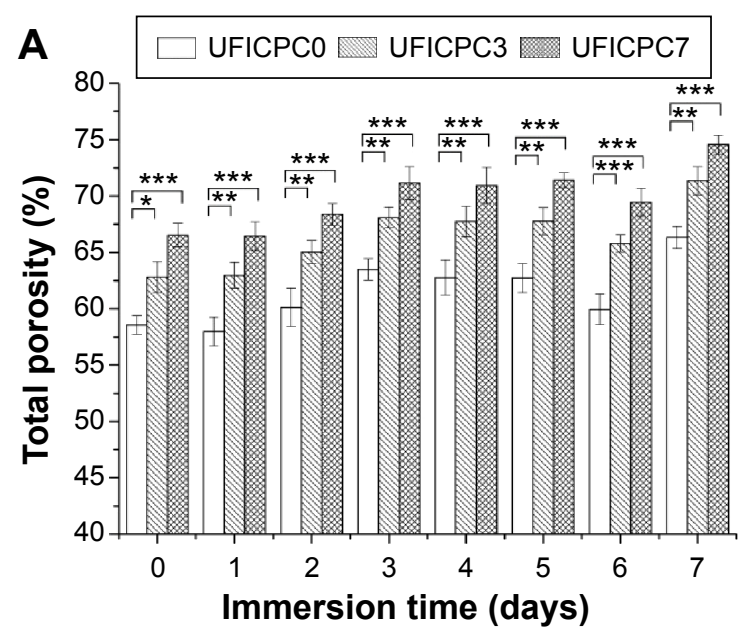

\section{Histological analysis}

Representative histological images are shown in Figure 8. Bone apposition on the pristine CPC cement (UFICPC0, Figure 8A) surface without any fibrous encapsulation was shown in three periods. Fibrous encapsulation occurred for all ultrafine fiber-incorporated samples (UFICPC3 and UFICPC7, Figure 8B and C). Moreover, the peripheral area of UFICPC7 showed an apparent fracture, as characterized by an irregular surface contour of the composite material. Compare to pristine UFICPC0, plenty of inflammatory cells have been found at the interface of tissue and composite (UFICPC3 and UFICPC7) in Figure 8B and C.

At 4 weeks after implantation, pristine CPC samples (UFICPC0, Figure 8Aa) revealed a more dense structure than

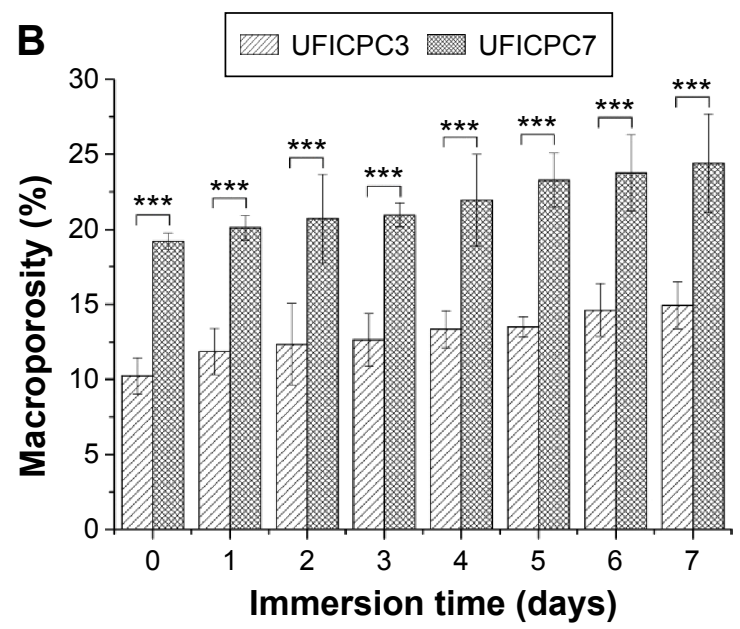

Figure 5 Total porosity (A) and macroporosity (B) of UFICPC0, UFICPC3, and UFICPC7 at different immersion times.

Notes: Before and after fiber degradation, total porosity of fiber-incorporated composites was statistic difference higher than pristine CPC cement (UFICPC0). Macroporosity of composites (both UFICPC3 and UFICPC7) also increased with increasing immersion time. Significantly different at $* P<0.05$, $* * P<0.01, * * * P<0.001$.

Abbreviations: CPC, calcium phosphate cement; UFICPC, ultrafine fiber-incorporated CPC. 


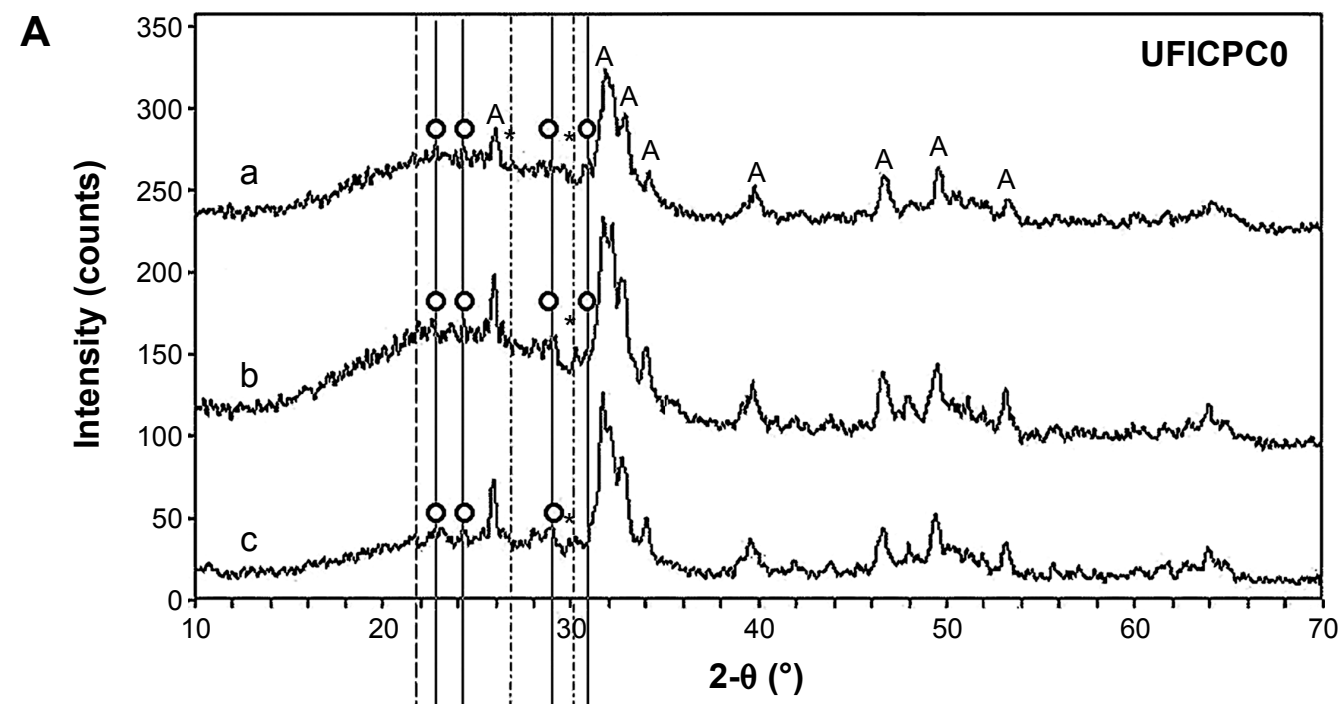

B

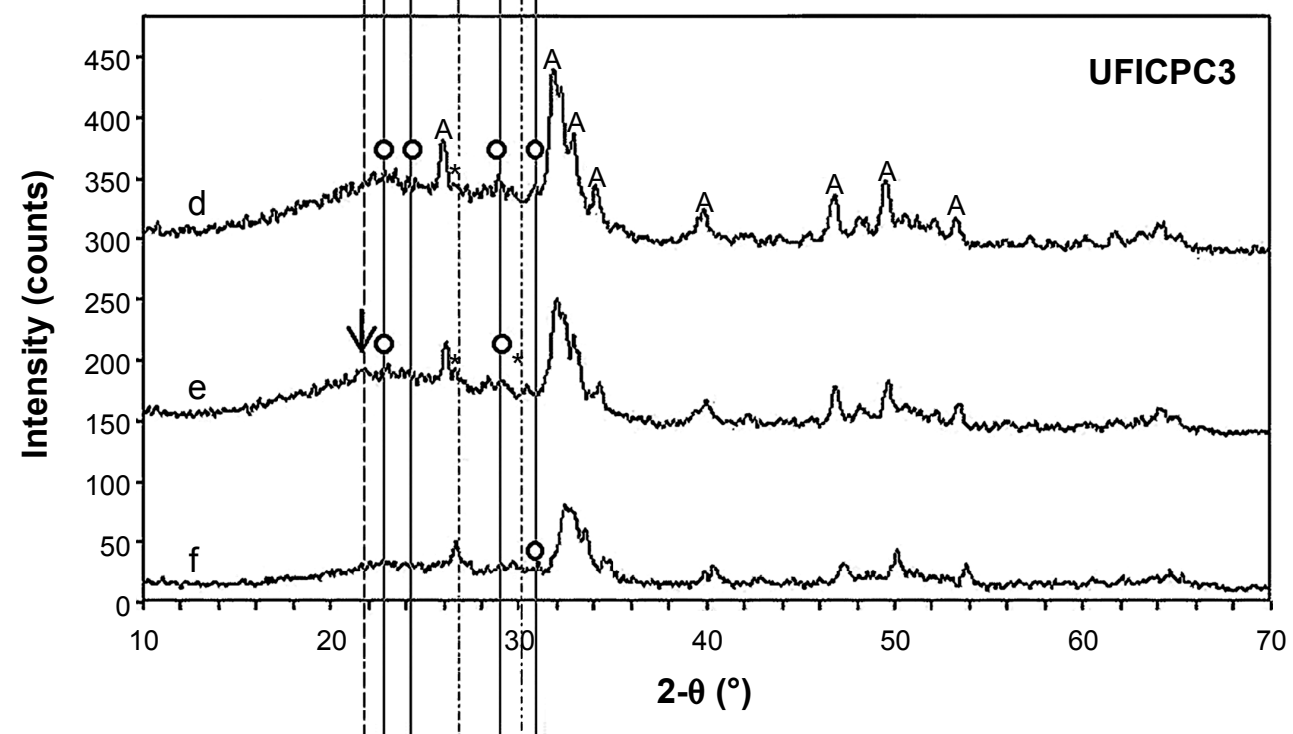

C

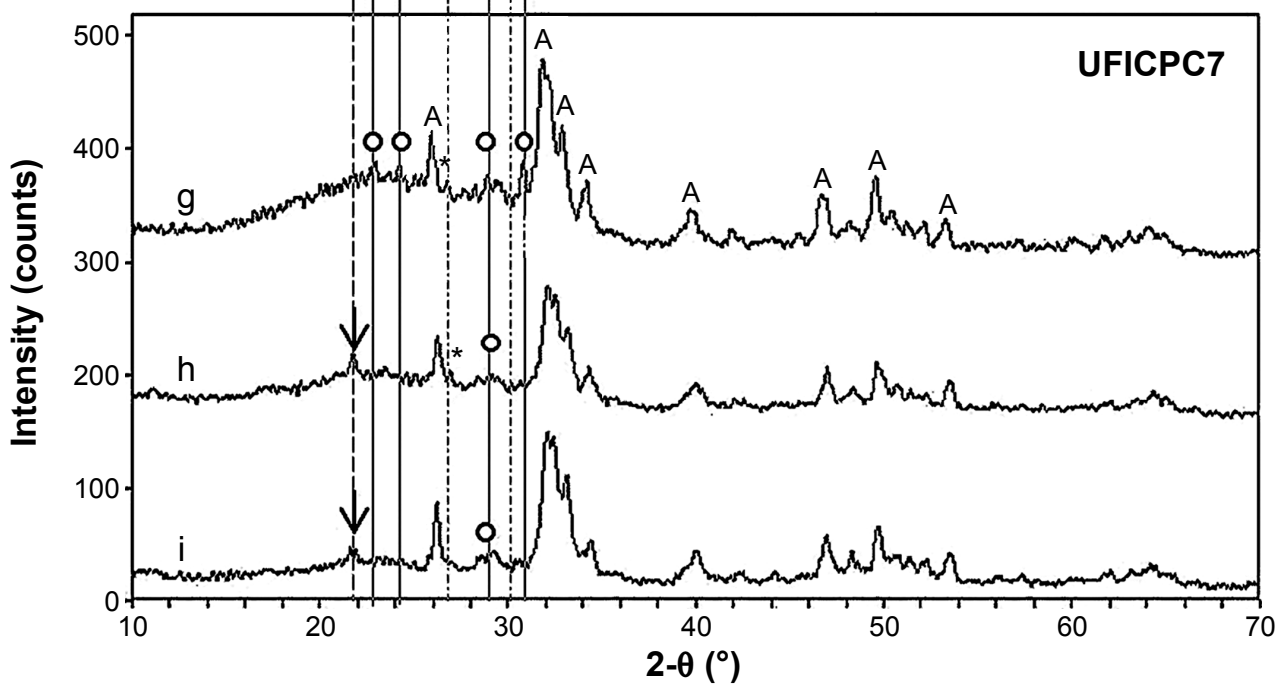

Figure 6 XRD patterns of UFICPC composites before (a, d, g) and after immersion in enzymatic PBS for I day (b, e, h) and 7 days (c, f, i): (a-c) for UFICPCO (A), (d-f) for UFICPC3 (B), and (g-i) for UFICPC7 (C).

Notes: The monetite and $\alpha$-TCP phase composition of fiber-incorporated composites (B and $\mathbf{C})$ disappeared and converted to apatite phase faster than pristine CPC cement (A). A: apatite, *: monetite, o: $\alpha-T C P$, arrow: PCL.

Abbreviations: CPC, calcium phosphate cement; PBS, phosphate buffer solution; PCL, poly( $\varepsilon$-caprolactone); TCP, tricalcium phosphate; UFICPC, ultrafine fiber-incorporated CPC; XRD, X-ray diffraction. 
A

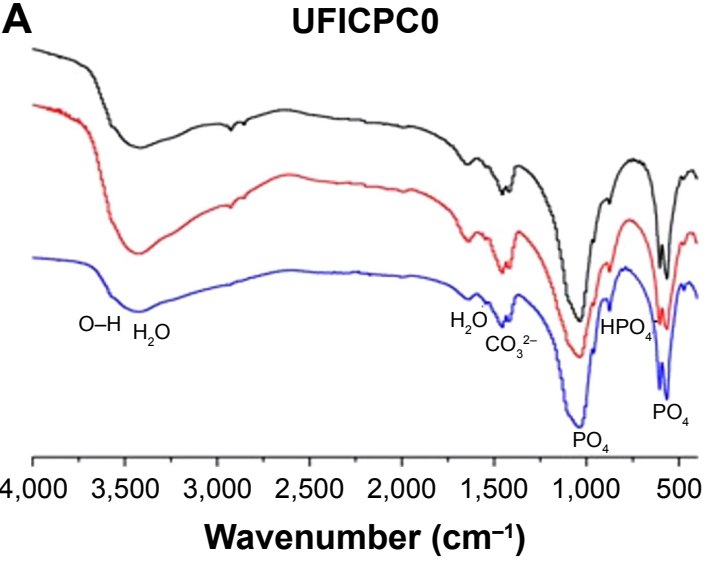

B

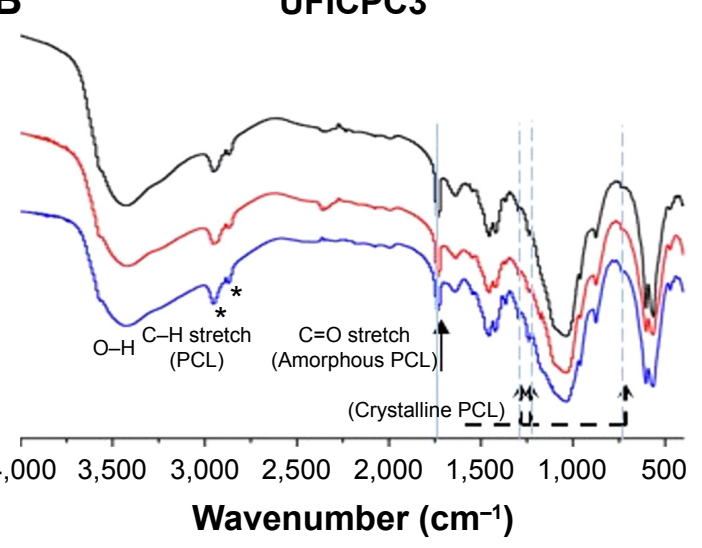

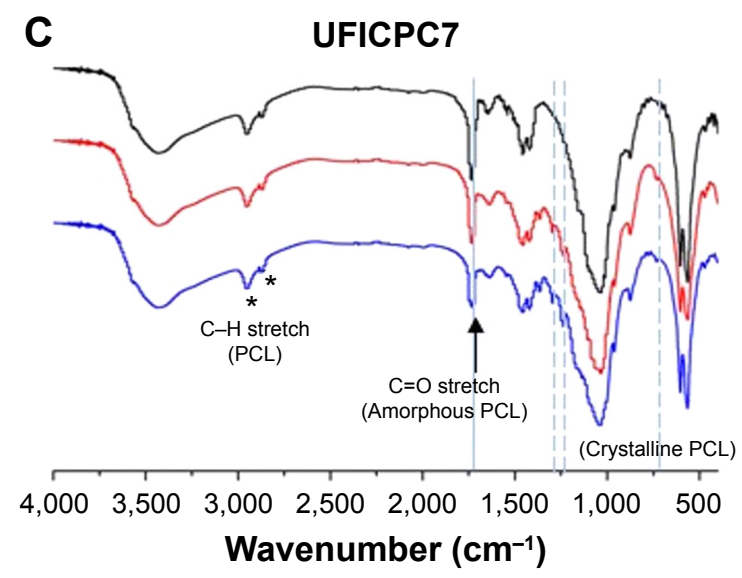

0 day -1 day 7 days

Figure 7 IR spectra of UFICPC composites before and after immersion in enzymatic PBS.

Notes: (A) UFICPC0, (B) UFICPC3, and (C) UFICPC7. Solid line represents the amorphous PCL peak, while dashed lines represent crystalline PCL peaks. For UFICPC3 and UFICPC7 composites, amorphous PCL peak decreased and crystalline PCL peaks increased apparently during incubation time. *indicates C-H stretch of PCL; black arrow indicates $\mathrm{C}=\mathrm{O}$ stretch of amorphous $\mathrm{PCL}$; dashed line indicates crystalline $\mathrm{PCL}$.

Abbreviations: CPC, calcium phosphate cement; IR, infrared; PBS, phosphate buffer solution; PCL, poly( $(\varepsilon$-caprolactone); UFICPC, ultrafine fiber-incorporated CPC.

other two composites (UFICPC3 and UFICPC7, Figure 8Bb and $\mathrm{Cc}$ ). At 8 weeks, the sample diameter of pristine $\mathrm{CPC}$ cement (Figure 8Ad) has no remarkable change in comparison with the shrinking area of UFICPC3 and UFICPC7 (Figure $8 \mathrm{Be}$ and $\mathrm{Cf}$ ). With increasing time, thicker fibrous capsule has been observed, as shown in Figure $8 \mathrm{~B}$ and $\mathrm{C}$ (UFICPC3 and UFICPC7) with more composites degraded. More broken pieces were separated from composite bulk by fibrous tissue (FT) and bone-like tissue ingrowth. At 12 weeks, the size of the all three samples becomes smaller, but the degradation happened inside of samples UFICPC3 (Figure $8 \mathrm{Bh}$ and $\mathrm{Ci}$, vs $\mathrm{Ag}$ ) and UFICPC7 in contrast to the peripheral areas of UFICPC0. Notably, fibrous capsule occupied the channel-like area and more new bone (NB) invaded into the cement gaps. For samples UFICPC 3 and UFICPC7 (Figure 8Bh and Ci), some divided fragments disappeared when bone-like tissue weaved in the degraded area. The observation and definition of bone-like tissue were referenced from similar histological phenomena in previous studies. ${ }^{19,20}$

The histological results indicate that fiber-incorporated CPC composites have faster degradation properties and more bone-like tissue fabrication interior compared to the pristine CPC cement. FT and NB incised and encompassed the cement remnants, which have been presented in Figure 9, at 12 weeks. Composite (C) was surrounded by a lot of macrophages (M, yellow arrows). As expected, multinucleated giant cells (yellow dash arrow) appeared at the surface of the composite, meaning a phagocytic process happened.

\section{Micro-CT reconstruction and quantification}

Micro-CT images were utilized for reconstruction and quantification of material degradation. The reversed 3D porous structure reconstructed by the shadow (holes and fiber bundles) of each sample is shown in Figure 10A-C. 


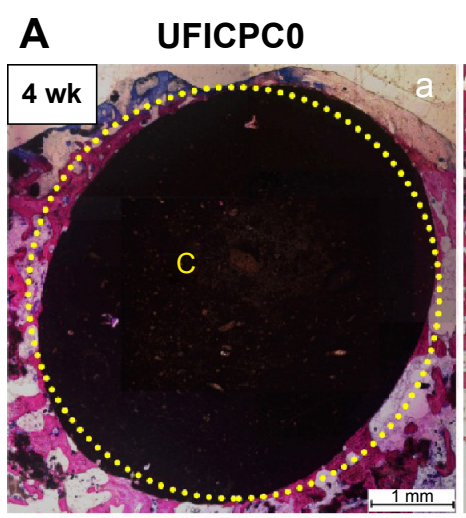

B UFICPC3

\section{UFICPC7}
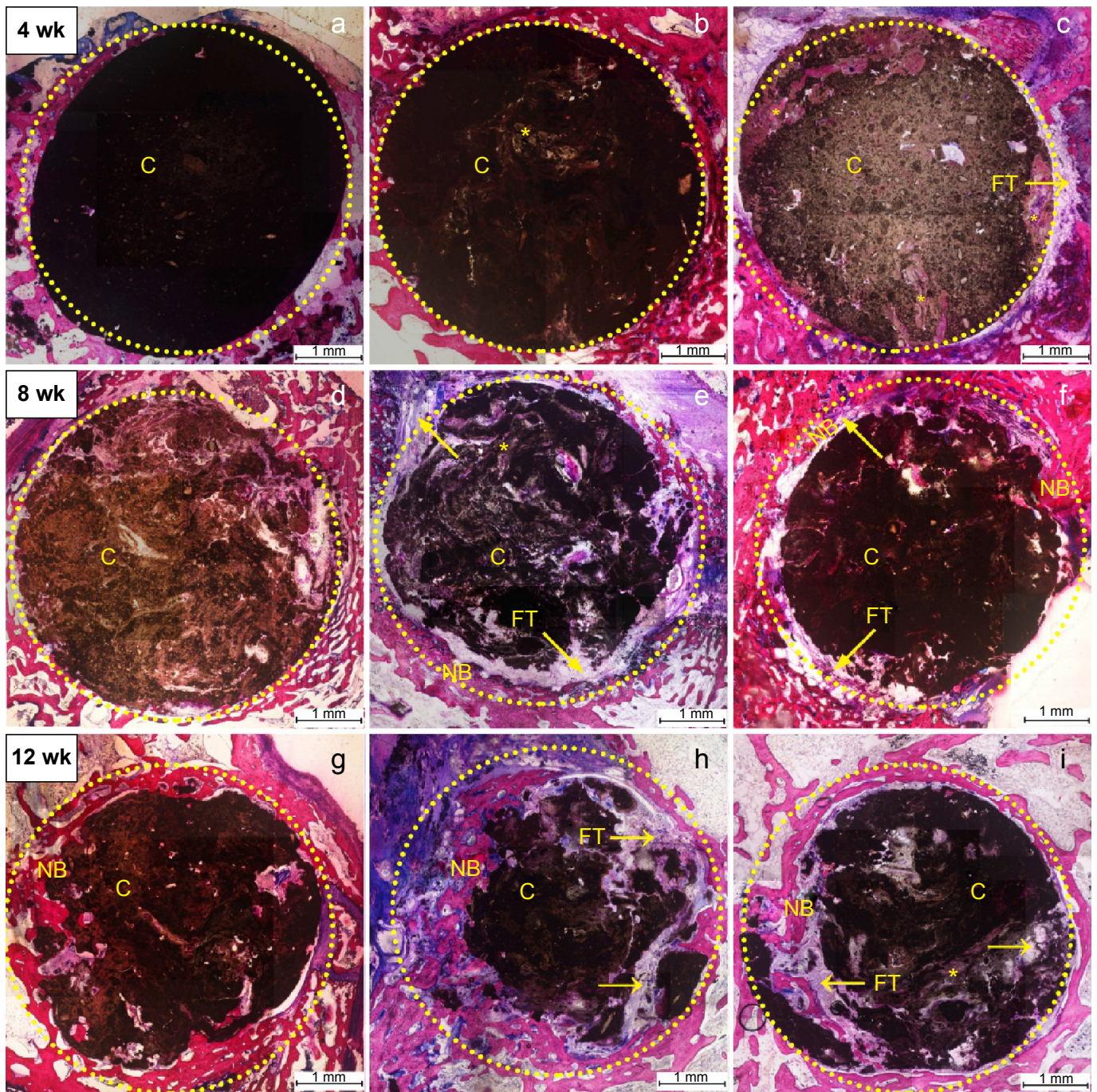

Figure 8 Representative histological section of three UFICPC composites, (A) UFICPCO, (B) UFICPC3 and (C) UFICPC7, after implantation for 4 (a-c), 8 (d-f) and 12 weeks $(\mathrm{g}-\mathrm{i})$

Note: Dotted circle covers original area of samples $(\Phi 5 \mathrm{~mm})$, arrow $(\rightarrow)$ indicates the fibrous capsule wall, and *shows the ultrafine PCL fibers.

Abbreviations: C, composite; CPC, calcium phosphate cement; FT, fibrous tissue; NB, new bone; PCL, poly( $\varepsilon$-caprolactone); UFICPC, ultrafine fiber-incorporated CPC; wk, weeks.
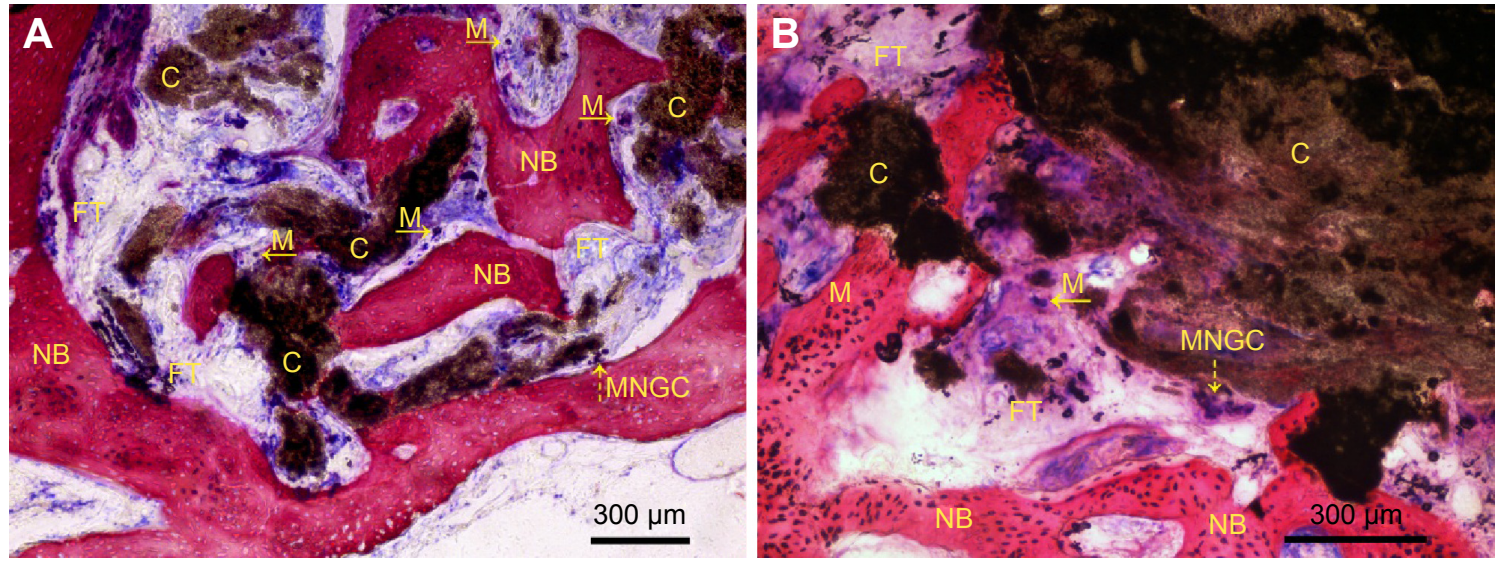

Figure 9 Representative histological section of the fiber-reinforced composites after 12 weeks on implantation.

Notes: (A) Ingrowth of NB into degraded composite was visible. Composite surrounded by macrophages (yellow arrows). (B) A multinucleated giant cell (yellow dashed arrow) appears at the surface of the composite which also has been visualized in (A). Yellow arrows indicate macrophages; Yellow dashed arrow indicates a multinucleated giant cell. Abbreviations: C, composite; FT, fibrous tissue; M, macrophage; MNGC, multinucleated giant cell; NB, new bone. 
Compared to sample UFICPC0 (Figure 10A) with many small pores (black dots) inside and some larger pores (green, yellow, and red area) on the surface, samples UFICPC3 (Figure 10B) and UFICPC7 (Figure 10C) exhibit more and larger pore volume and interconnected channel-like pores throughout the shell and core due to the incorporated ultrafine PCL fibers or fiber bundles.

After 24 weeks of implantation, only the surface of pristine CPC cement is surrounded by bone tissue (blue color), the CPC degradation is slow and limited to the peripheral area. The sample still keeps its original shape and relatively dense structure, nearly no bone formation presents in its body, as shown in Figure $10(\mathrm{D}, \mathrm{G})$. However, the cement bodies of UFICPC3 (Figure 10E, H) and UFICPC7 (Figure 10F, I) become incomplete, and degraded area appears both on the outer surface and within their body, which is occupied by the NB (blue color). The formed bone network is mainly attributed to the fast degradation of ultrafine PCL fibers or
UFICPCO

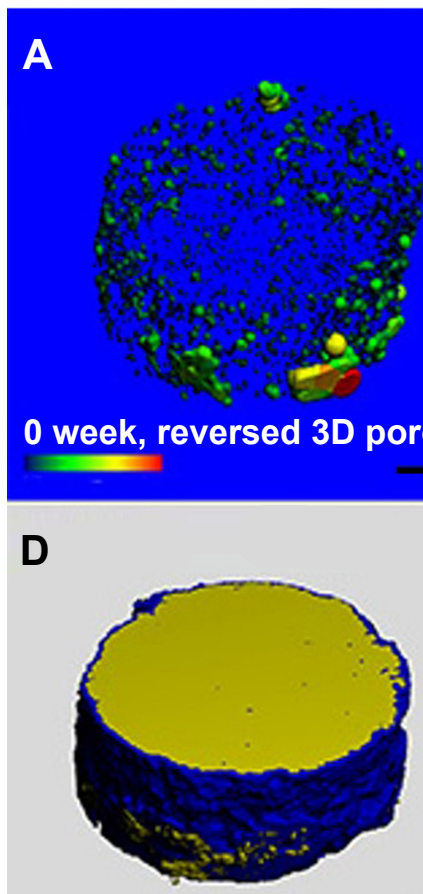

UFICPC3

B

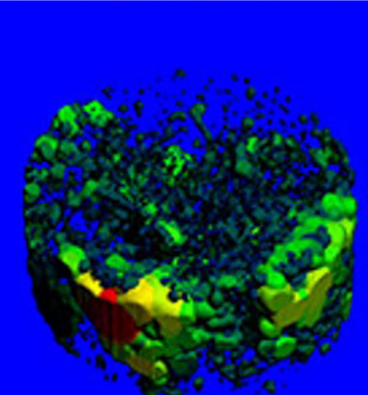

UFICPC7

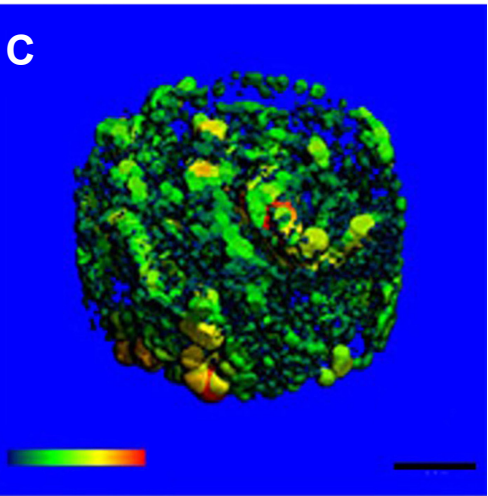

$\mathbf{F}$

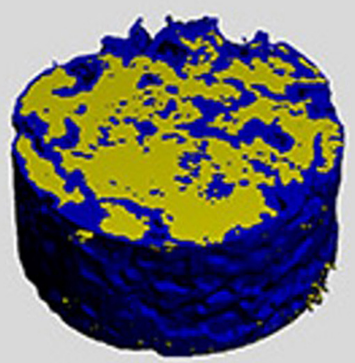

24 weeks, samples with surrounding bone

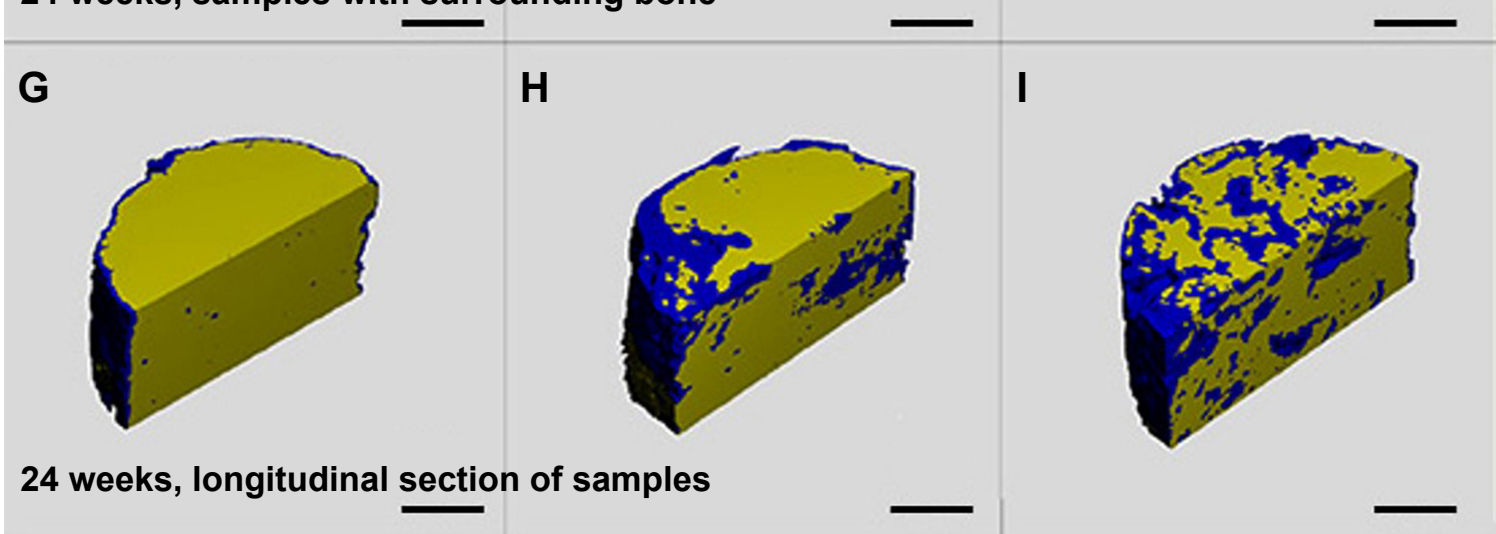

Figure 10 3D micro-CT reconstruction and 3D longitudinal section models before and after implantation.

Notes: 3D micro-CT reconstruction models of the reversed 3D porous structure without CPC matrix before implantation (A-C). UFICPC3 and UFICPC7 exhibited more and larger pore volume and interconnected channel-like pores throughout the shell and core. Also shown are 3D micro-CT reconstruction models of cements after implantation for 24 weeks (D-F) and 3D longitudinal section models of cements after implantation for 24 weeks (G-I). The pristine CPC degradation was slow and the bone regeneration was limited to the peripheral area $(\mathbf{D}, \mathbf{G})$. On the other hand, the cement bodies of UFICPC3 (E, H) and UFICPC7 (F, I) were fragmented, and the new bone (blue color) occupied both on the outer surface and within their body. Bar: I mm.

Abbreviations: CPC, calcium phosphate cement; CT, computed tomography; UFICPC, ultrafine fiber-incorporated CPC; 3D, three dimensional. 
Table 2 Bone (BV/TV), material (MV/TV) volume fraction, and trabecular number (Tb. N) calculated from micro-CT after 24 weeks of implantation

\begin{tabular}{llll}
\hline Sample & \multicolumn{3}{l}{ 24 weeks after implantation } \\
\cline { 2 - 4 } & BV/TV (\%) & MV/TV (\%) & Tb. N ( I/mm) \\
\hline UFICPC0 & 6.31 & 84.39 & 2.03 \\
UFICPC3 & 22.66 & 68.86 & 2.85 \\
UFICPC7 & 23.66 & 67.05 & 3.34 \\
\hline
\end{tabular}

Note: Both BV/TV fraction and trabecular number of UFICPC3 and UFICPC7 samples were higher than pristine CPC cement (UFICPCO).

Abbreviations: $\mathrm{BV}$, bone volume; $\mathrm{CPC}$, calcium phosphate cement; $\mathrm{CT}$, computed tomography; MV, material volume; Tb. N, trabecular number; TV, tissue volume; UFIPC, ultrafine fiber-incorporated CPC.

fiber bundles that leave behind abundant interconnected grooves and channels.

The fractions of BV/TV, MV/TV, and Tb. $\mathrm{N}$ from tomographic data of micro-CT are listed in Table 2. Compared to pristine CPC cement, the composites UFICPC 3 and UFICPC7 cements showed higher BV/TV fraction and TV, as well as lower MV/TV fraction.

\section{Discussion}

This work aimed to characterize the effect of the ultrafine PCL fibers on the in vitro and in vivo degradation as well as bone regeneration of CPC/PCL composites. Electrospun PCL fibers were collected by water pool and fabricated as bundles with nano to micro fibrous structure, as shown in Figure 2A. Two different weight percentages of ultrafine PCL fibers (3\% and $7 \%$ ) were incorporated into CPC matrix.

$\mathrm{CPC}$ is an osteoconductive and injectable material for filling of bone defects. However, the intrinsic porosity of pristine CPC after setting is too small for bone ingrowth. ${ }^{21}$ The introduction of macro- and microporosity within CPC can be used to promote CPC degradation and bone ingrowth. Therefore, two different strategies can be used to obtain porous CPC cements, ie, the instantaneous or delayed approach. ${ }^{22}$ Instantaneous porous $\mathrm{CPC}$ involves the creation of $\mathrm{CO}_{2}$ bubbles during setting, whereas delayed porous $\mathrm{CPC}$ is obtained by the incorporation of degradable polymeric microspheres or fibers into the CPC matrix. In the instantaneous foaming method, the pores created by upward migration of gas bubbles during setting cannot be controlled in terms of size and distribution. ${ }^{23}$ Incorporation of PLGA or gelatin microspheres in CPC cements results in the production of a controlled macro/microporosity during in situ degradation of the microspheres. ${ }^{15}$ However, the inclusion of PLGA microspheres is associated with a loss of mechanical strength of the set cement. Incorporated CPC with degradable fibers can be a major strategy to overcome the present limitations of CPC. ${ }^{1}$
Absorbable fiber mesh, fiber mat, or suture fibers, like polyglactin $910,9,24-26$ chitosan fiber, ${ }^{27,28}$ PLGA fibers, ${ }^{9}$ and Vicryl (a copolymer of glycolic acid and lactic acid), ${ }^{29}$ have been introduced into CPC cements to create macroporosity. However, a fiber content greater than $2-3$ vol\% results in poor workability and fiber dispersion of the cement paste. ${ }^{1}$ In view of this, electrospinning has been extensively explored as a method for the fabrication of nano- or micrometer fibers. Thinner diameter of fibers will provide a larger interface area between fibers and cement matrix for a given fiber volume fraction. As confirmed by our incubation assay, the subsequent porosity of CPC, which contains such ultrafine fibers, can indeed significantly be enhanced by the degradation of the incorporated fibers. After 7 days of incubation, a twofold macroporosity has been developed by the lipase catalyzed PCL degradation for UFICPC7 specimen, which was $24.5 \%$ vs the calculated fiber volume (12.29\%). It can be also seen from the images in Figures 2C, D, 3E-L, and the reversed 3D porous structure in Figure 10B, C of composite cements that the interconnected fibrous structure have been formed in the fiber-incorporated cement matrix.

From a material point of view, PCL may not be a preferable degradable material like PLGA due to its long-term degradability. ${ }^{30}$ Despite this, electrospun fibers of PCL and PLGA have been visualized to have different morphologies. According to our previous study, ${ }^{13}$ ultrafine PCL fibers gathered as a bundle-like fibrilia but ultrafine PLGA fibers aggregated as a cotton-like material. The former is apparently easier to disperse into CPC matrix than the latter. In addition, a linear increase of weight loss with time has been found for both pure PCL fibers and the fiber composite cements (Figure 4), similar to the report of lipase catalyzed enzymatic degradation of PCL. ${ }^{31}$ Compared to PCL bulk, ${ }^{32}$ the degradability can be improved by increasing the interface of ultrafine PCL fibers. Fibrous PCL degrades through random hydrolytic chain scission of the ester linkage, which was confirmed by the decrease of the amorphous peak at $1,729 \mathrm{~cm}^{-1}$ in the FTIR spectra (Figure 7). Thinner PCL fibers with a diameter from nano to micro scale become water-soluble and participate in the weight loss, larger body of this polymer require more scission steps to become degraded into sufficiently short fragments. This trend is nicely confirmed by monolayer degradation experiments. ${ }^{31,33}$ The incorporation and degradation of the ultrafine PCL fibers also apparently influenced the cement phase in vitro conversion. The weak acidic $\mathrm{pH}$ value, as caused by PCL degradation, can support the rapid hydrolytic conversion of monetite and $\alpha$-TCP into HA phase (Figure 6), leading to a fast hardening of the $\mathrm{CPC}$ cement. ${ }^{34}$ 
The in vitro data and in vivo results confirmed that indeed the incorporation of electrospun PCL fibers promoted the degradation of the CPC cement and preliminary bone ingrowth. After in vitro immersion in lipase PBS, the grooves, and interconnected channels (Figure 3), the total porosity and macroporosity (Figure 5) dramatically increase with time in the ultrafine fiber-incorporated cement matrix. According to the data and images by micro-CT, the fraction of BV of sample UFICPC7 reaches $23.66 \%$ after 24 weeks of implantation (Table 2), and the degraded space has been occupied by a lot of newly formed bone (Figures $8-10$ ). The fraction of $\mathrm{BV}$ of the two fiber-incorporated $\mathrm{CPC}$ composites (UFICPC3 and UFICPC7) was $~ 3.5$ times higher than that of the pristine CPC (UFICPC0, only 6.31\%).

Moreover, higher weight fraction of degradable fibers was more efficient in macropore formation and tissue infiltration. From longitudinal section of samples after 24-week implantation, more bone-like tissue (blue color) was observed inside the core of UFICPC7 sample (Figure 10I) than that of UFICPC3 (Figure 10H). The biological results in vivo matched the faster degradation (Figure 4) and higher macroporosity (Figure 5) in vitro. Both of them originated in higher fiber addition of UFICPC7.

Unfortunately, the degradation of PCL fiber in CPC matrix still caused some acidic problem in vivo even at $\mathrm{pH}$ value of 6.5 , while testing for enzymatic degradation in vitro on day 7 . Compared to UFICPC3, more inflammatory cells have been observed for more acidic products degraded from UFICPC7 samples (Figures 1B and 8). The amount of inflammatory cell infiltration and fibrous capsules have also been observed with the increasing implantation time. Normally, polycaprolactone is subjected to biodegradation as random hydrolytic chain scission of the ester linkage, and the products are either metabolized via the tricarboxylic acid cycle or eliminated by direct renal secretion. ${ }^{35}$ In this study, the acidic degradation products from PCL did not get completely neutralized by the soluble calcium ions of CPC according to our design. The hypothetic reason could be faster diffusion or concentration of acidic products. The former may be caused by more fiber dispersed in the surface of the UFICPC7 samples, and the latter always root from in situ degradation of thicker intertwined fibers (seen in the micro-CT cross sections). Thus, the degradation of fibers resulted in gathering of higher partial acidic products, which led to the acute inflammatory reaction and fibrous capsule hyperplasia, as shown in Figure 8. The same inherent cytotoxicity as potential inflammatory and foreign body reaction responses has also been discovered in similar types of systems as PLGA systems. ${ }^{30}$ Therefore, we should think about how to mix higher ratio of the ultrafine fibers or bundles into CPC cement to fabricate a homogenous composite, which could provide a more biofriendly environment and better interconnective porous structure for cells and tissue ingrowth in the next step.

It is noteworthy to mention that the ultrafine degradable fibers also assist the load of cytokines or drugs, by enabling a mild preparation process for drug delivery application. ${ }^{12}$ The functional fiber-incorporated cement composites would promote more efficient tissue regeneration and bone reconstruction in future.

\section{Conclusion}

Electrospun PCL fibers and fiber bundles have been incorporated into CPC matrix, and the effects of ultrafine PCL fibers on the degradability in vitro and in vivo were investigated. The incorporation of nano- to micrometer fibers significantly increased the macroporosity of cements and provided larger degraded space for bone formation and ingrowth than the pristine CPC cement. The in vitro and in vivo results demonstrate a promising method to use degradable ultrafine fibers in preparation of $\mathrm{CPC}$ bone cements or bone tissue engineering scaffolds.

\section{Acknowledgments}

The authors appreciate financial support from National 863 Project (No 2015AA033702), China NSFC project (31370971), and China-Netherlands PSA project (2008DFB50120 and PSA 08-PSA-M-02 [Dutch]).

\section{Disclosure}

The authors report no conflicts of interest in this work.

\section{References}

1. Krüger R, Groll J. Fiber reinforced calcium phosphate cements-on the way to degradable load bearing bone substitutes? Biomaterials. 2012;33: 5887-5900.

2. Sowmya S, Bumgardener JD, Chennazhi KP, Nair SV, Jayakumar R. Role of nanostructured biopolymers and bioceramics in enamel, dentin and periodontal tissue regeneration. Prog Polym Sci. 2013;38(10-11): 1748-1772.

3. Dorozhkin SV. Calcium orthophosphate cements for biomedical application. J Mater Sci. 2009;44:2343-2387.

4. Zhang JT, Liu WZ, Schnitzler V, Tancret F, Bouler JM. Calcium phosphate cements for bone substitution: chemistry, handling and mechanical properties. Acta Biomater. 2014;10(3):1035-1049.

5. Ooms EM, Wolke JGC, van der Waerden JPCM, Jansen JA. Trabecular bone response to injectable calcium phosphate $(\mathrm{Ca}-\mathrm{P})$ cement. J Biomed Mater Res. 2002;61(1):9-18.

6. Fullana G, Ternet H, Freche M, Lacout JL, Rodriguez F. Controlled release properties and final macroporosity of a pectin microspheres-calcium phosphate composite bone cement. Acta Biomater. 2010;6:2294-2300.

7. Canal C, Ginebra MP. Fibre-reinforced calcium phosphate cements: a review. J Mech Behav Biomed Mater. 2011;4:1658-1671. 
8. Wahwah TH, Hockin HKX. Collagen-calcium phosphate cement scaffolds seeded with umbilical cord stem cells for bone tissue engineering. Tissue Eng Part A. 2011;17(23-24):2943-2953.

9. Kim JK, McBride S, Fulmer M, et al. Fiber-reinforced calcium phosphate cement formulations for cranioplasty applications: a 52-week duration preclinical rabbit calvaria study. J Biomed Mater Res Part B Appl Biomater. 2012;100B:1170-1178.

10. Guo DG, Sun HL, Xu KW, Hang Y. Long-term variations in mechanical properties and in vivo degradability of CPC/PLGA composite. J Biomed Mater Res B Appl Biomater. 2007;82B:533-544.

11. Holzwarth JM, Ma PX. Biomimetic nanofibrous scaffolds for bone tissue engineering. Biomaterials. 2011;32:9622-9629.

12. Huang ZM, Zhang YZ, Kotaki M, Ramakrishna S. A review on polymer nanofibers by electrospinning and their applications in nanocomposites Compos Sci Technol. 2003;63:2223-2253.

13. Zuo Y, Yang F, Wolke JG, Li YB, Jansen JA. Incorporation of biodegradable electrospun fibers into calcium phosphate cement for bone regeneration. Acta Biomater. 2010;6(4):1238-1247.

14. Reiche KJ, Hartmann J, Kratz K, Lendlein A. Selective enzymatic degradation of poly( $\varepsilon$-caprolactone) containing multiblock copolymers. Eur J Pharm Biopharm. 2008;68:45-46.

15. Habraken WJEM, Zhang Z, Wolke JGC, et al. Introduction of enzymatically degradable poly(trimethylene carbonate) microspheres into an injectable calcium phosphate cement. Biomaterials. 2008;29: 2464-2476.

16. Gauthier O, Müller R, Stechow D, et al. In vivo bone regeneration with injectable calcium phosphate biomaterial: a three-dimensional microcomputed tomographic, biomechanical and SEM study. Biomaterials. 2005;26:5444-5453.

17. Wen CY, Qin L, Lee KM, Chan KM. The use of brushite calcium phosphate cement for enhancement of bone-tendon integration in an anterior cruciate ligament reconstruction rabbit model. J Biomed Mater Res B Appl Biomater. 2009;89B:466-474.

18. Habraken WJEM, Liao HB, Zhang Z, et al. In vivo degradation of calcium phosphate cement incorporated into biodegradable microspheres. Acta Biomater. 2010;6:2200-2211.

19. Liao HB, Walboomers XF, Habraken WJEM, et al. Injectable calcium phosphate cement with PLGA, gelatin and PTMC microspheres in a rabbit femoral defect. Acta Biomater. 2011;7:1752-1759.

20. Grover LM, Wright AJ, Gbureck U, et al. The effect of amorphous pyrophosphate on calcium phosphate cement resorption and bone generation. Biomaterials. 2013;34:6631-6637.

21. Espanol M. Intrinsic porosity of calcium phosphate cements and its significance for drug delivery and tissue engineering applications. Acta Biomater. 2009;5:2752-2762.
22. Klijn RJ, van den Beucken JJJP, Lanao RPF, et al. Three different strategies to obtain porous calcium phosphate cements: comparison of performance in a rat skull bone augmentation model. Tissue Eng Part A. 2012;18(11-12):1171-1181.

23. Bodde EW, Cammaert CT, Wolke JG, Spauwen PH, Jansen JA. Investigation as to the osteoinductivity of macroporous calcium phosphate cement in goats. J Biomed Mater Res B Appl Biomater. 2007;83:161-168.

24. Zhou HZ, Weir MD, Xu HHK. Effect of cell seeding density on proliferation and osteodifferentiation of umbilical cord stem cells on calcium phosphate cement-fiber scaffold. Tissue Eng Part A. 2011;17(21-22): 2603-2613.

25. Xu HHK, Quinn JB, Takagi S, Chow LC. Synergistic reinforcement of in situ hardening calcium phosphate composite scaffold for bone tissue engineering. Biomaterials. 2004;25:1029-1037.

26. Lee $\mathrm{K}$, Weir MD, Lippens E, et al. Bone regeneration via novel macroporous CPC scaffolds in critical-sized cranial defects in rats. Dent Mater. 2014;30:e199-e277.

27. Lian Q, Li DC, Jin ZM, Wang J. Fabrication and in vitro evaluation of calcium phosphate combined with chitosan fibers for scaffold structures. J Bioact Compat Polym. 2009;24:113-124.

28. Pan ZH, Jiang PJ. Assessment of the suitability of a new composite as a bone defect filler in a rabbit model. J Tissue Eng Regen Med. 2008;2 347-353.

29. Chen WC, Zhou HZ, Weir MD, Bao CY, Xu HHK. Umbilical cord stem cells released from alginate-fibrin microbeads inside macroporous and biofunctionalized calcium phosphate cement for bone regeneration. Acta Biomater. 2012;8:2297-2306.

30. Anderson JM, Shive MS. Biodegradation and biocompatibility of PLA and PLGA microspheres. Adv Drug Deliv Rev. 2012;64:72-82.

31. Kulkarni A, Reiche J, Hartmann J, Kratz K, Lendlein A. Selective enzymatic degradation of poly( $\varepsilon$-caprolactone) containing multiblock copolymers. Eur J Pharm Biopharm. 2008;68:46-56.

32. Sun HF, Mei L, Song CX, Cui XM, Wang PY. The in vivo degradation, absorption and excretion of PCL-based implant. Biomaterials. 2006;27:1735-1740

33. Reiche J, Kulkarni A, Kratz K, Lendlein A. Enzymatic monolayer degradation study of multiblock copolymers consisting of poly ( $\varepsilon$-caprolactone) and poly(p-dioxanone) blocks. Thin Solid Films. 2008; 516(24):8821-8828.

34. Ginebra MP, Traykova T, Planell JA. Calcium phosphate cements as bone drug delivery systems: a review. J Control Release. 2006;113: $102-110$.

35. Kweon HY, Yoo MK, Park IK, et al. A novel degradable polycaprolactone networks for tissue engineering. Biomaterials. 2003;24:801-808.
International Journal of Nanomedicine

\section{Publish your work in this journal}

The International Journal of Nanomedicine is an international, peerreviewed journal focusing on the application of nanotechnology in diagnostics, therapeutics, and drug delivery systems throughout the biomedical field. This journal is indexed on PubMed Central, MedLine, CAS, SciSearch $®$, Current Contents $\AA /$ Clinical Medicine,

\section{Dovepress}

Journal Citation Reports/Science Edition, EMBase, Scopus and the Elsevier Bibliographic databases. The manuscript management system is completely online and includes a very quick and fair peer-review system, which is all easy to use. Visit http://www.dovepress.com/ testimonials.php to read real quotes from published authors. 